\title{
Effect of N-95 Mask on Oxygen Saturation Level in Health Care Workers and Their Experience While Using N-95 Mask
}

\author{
Devika Sinha ${ }^{1}$ Satheesh Kumar Bhandary ${ }^{1}$ Rajeshwary Aroor ${ }^{1}$ Rashmitha Reddy ${ }^{1}$ Shravan Alva ${ }^{1}$ \\ ${ }^{1}$ Department of Otorhinolaryngology, KS Hegde Medical Academy, \\ Nitte (Deemed to be University), Mangalore, Karnataka, India \\ Address for correspondence Rajeshwary Aroor, MBBS, MS, \\ Department of ENT, KS Hegde Medical Academy, Nitte (Deemed to be \\ University), Mangalore 575018, Karnataka, India \\ J Health Allied Sci ${ }^{\mathrm{NU}}$ 2022;12:274-276. \\ (e-mail: rajeshwarisomayaji@gmail.com).
}

\begin{abstract}
Background N-95 masks are in high demand during this COVID-19 pandemic but wearing an $\mathrm{N}-95$ mask is a real challenge to the health workers during their duty hours. The present study aims to measure the effect of the $\mathrm{N}-95$ mask on oxygen saturation level in the blood and the discomforts experienced while using it.

Materials and Methods Oxygen saturation was measured in 80 health care workers before and after wearing the $\mathrm{N}-95$ mask continuously for more than 6 hours. The participants were asked to fill a questionnaire regarding various discomforts of $\mathrm{N}-95$

Keywords

- N-95 mask

- health care workers

- oxygen saturation

- $\mathrm{SpO}_{2}$

- COVID-19

- discomfort mask usage, which consisted of 14 questions.

Results The most common discomfort was pain around the pinna followed by difficulty in breathing and fogging of spectacles or goggles. In addition, $71 \%$ of them had decreased oxygen saturation by $1 \%$.

Conclusion $\mathrm{N}-95$ mask can alter the oxygen saturation but it does not produce significant effects in healthy individuals. It can significantly affect patients with compromised airways.
\end{abstract}

\section{Introduction}

The emergence of the COVID-19 pandemic caused by a novel strain of $\beta$-coronavirus in $2019^{1}$ led to an increased demand for personal protective equipment (PPE), especially the N-95 mask. It is mandatory for the health care workers who are at constant threat of contracting the disease. These masks prevent infection entry through oral and nasal routes. The number " 95 " indicates that it filters $95 \%$ of the particles of 0.3 microns size. ${ }^{2}$ A routine surgical mask is not much useful for health care workers during this COVID pandemic. It mainly prevents the spread of infectious droplets and aerosols into the air generated from the user of the mask. ${ }^{3}$

published online

December 30, 2021
DOI https://doi.org/ 10.1055/s-0041-1740329. ISSN 2582-4287.
The N-95 mask owing to its snug fit and seal created all around the oral and nasal passage leads to various kinds of discomforts among the users. Uneasiness and discomfort are the main drawbacks of the N-95 mask. Difficulty in breathing, headache, giddiness, generalized fatigue, and decreased concentration at work are other main complaints. ${ }^{4}$ All these problems could be attributed to the reduced oxygen availability to the user while breathing through the mask. When used for long working hours, these masks can cause decreased oxygen availability, leading to breathing difficulty, headache, and fatigue. In the initial period of the pandemic, scarcity of both health care workers and PPE kits forced the health care workers to work for a long duration of 6 to 8 hours. This creates

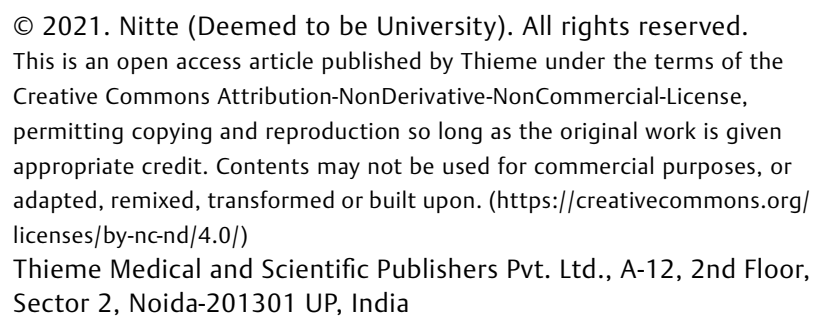


a lot of inconveniences, mental and physical strain on health care workers. The phobia of getting oneself infected further exaggerated the psychological and physical stress.

Very few studies are available in the literature describing the side effects of wearing an N-95 mask. But none of them measured oxygen saturation during mask usage in healthy health care workers. In this study, we tried to find out the oxygen saturation before and at the end of the N-95 mask use and common problems encountered by its prolonged use.

\section{Materials and Methods}

This is a prospective observational study done on 80 healthcare workers assigned to the COVID area, i.e., the fever clinic of a tertiary care hospital. The study duration was two months, from August 2020 to September 2020. Health care workers who were using N-95 masks for more than 6 hours continuously were included after informed consent and after institutional review board (IRB) approval. Out of 80 participants, 40 were males, and 40 were females. All participants underwent oxygen saturation estimation before using the $\mathrm{N}-95$ mask and again at the end of the duty before removing the mask.

During the duty hours, the participants were instructed not to remove the mask. All the participants were asked to fill a questionnaire regarding various discomforts of N-95 mask usage, which consisted of 14 questions and 1 open-ended question. They were asked if they had difficulty in breathing, itching over the face and mouth, rash on the face, pain at the back of the pinna, facial heat, facial pressure, headache, giddiness, generalized fatigue, frequent fogging of the spectacles/goggles, difficulty in communication, nasal discharge, nasal obstruction, and mouth breathing. They were also asked to score the efficacy of the $\mathrm{N}-95$ mask in protecting them from getting infected.

\section{Results}

The results revealed that $51 \%$ of the participants were familiar with the N-95 mask. The majority (67\%) did not get acclimatized to wearing the mask and continued to face the same discomforts. Pain around the pinna (70\%), difficulty in communication (58\%), fogging of glasses/spectacles (55\%), difficulty in breathing (50\%), itching over the face $(41 \%)$, and mouth breathing (33\%) were the most common complaints ( - Fig. 1A, B). Pain over the dorsum of the nose and frequent

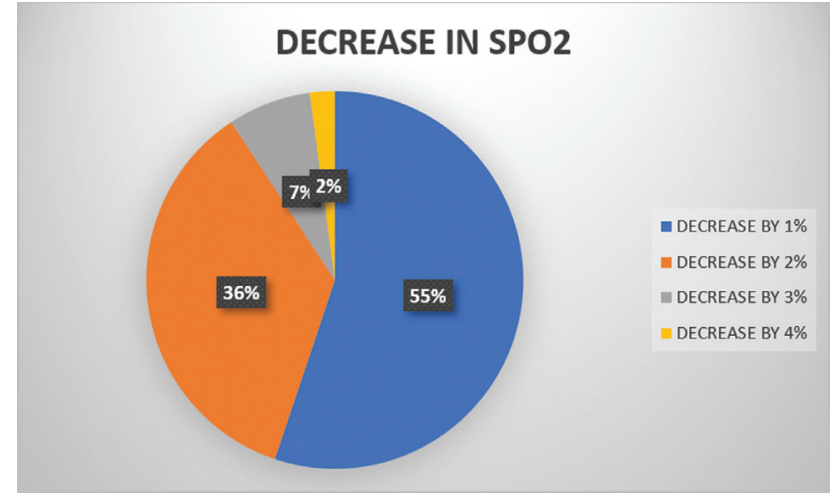

Fig. 2 Percentage decrease in $\mathrm{SpO}_{2}$ among participants who had a decrease in oxygen saturation level after duty.

thirst were some of the other reported complaints. The mean pretest oxygen saturation was $98.4 \%$, and the value at the end was $97.33 \%$. In addition, $71 \%$ of the participants showed a decrease in the blood oxygen saturation level, whereas the remaining $29 \%$ did not change. The majority of them had only a $1 \%$ decrease in oxygen saturation ( - Fig. 2). The majority of the participants (76\%) complained of decreased work efficiency at the end of 6 to 8 hours of duty after continuously wearing the mask. There was no statistical difference between 8-hour duty and 6-hour duty participants (-Table 1 ). The participants were asked to rate the effectiveness of the N-95 mask in protecting them from getting infected. The majority of them gave a score of 3 out of 5 . Those who did 8 hours of duty felt that they were more exhausted at the end of the duty than those who did 6 hours. Most participants felt that masks of variable sizes should be available to suit one's facial structure. People with a smaller facial structure felt the need to readjust the mask repeatedly as the mask kept becoming loose.

\section{Discussion}

The emergence of the COVID-19 pandemic and the morbidity and mortality associated with it created an emergency worldwide. The available health care facilities faced a sudden and humongous caseload of patients suspected or confirmed to have a coronavirus infection.

Personal protective equipment, especially the N-95 mask, played a crucial role in preventing viral entry through the
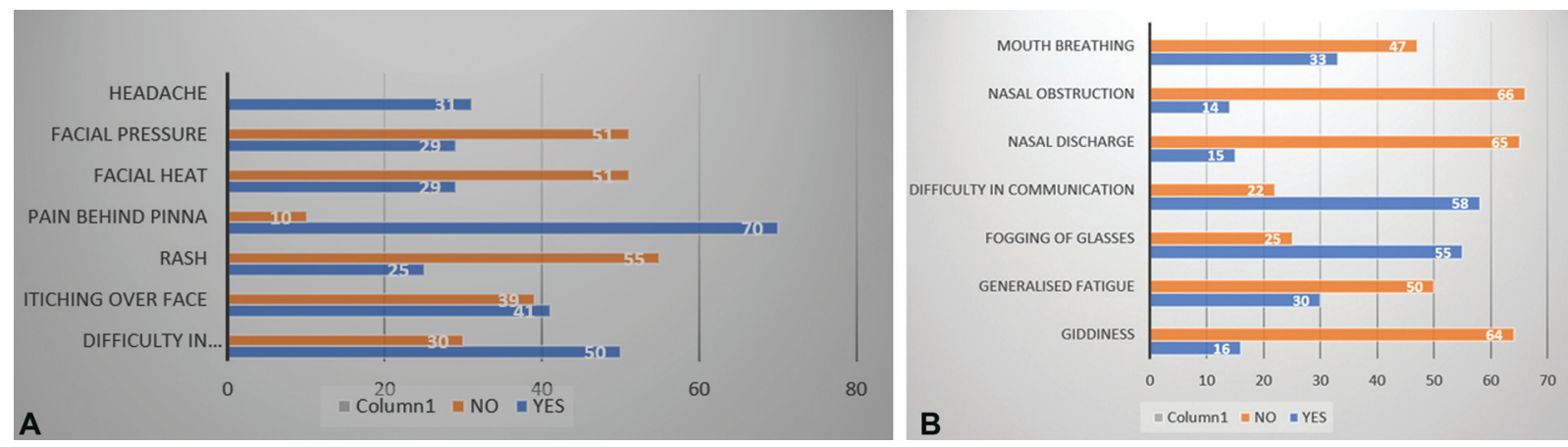

Fig. 1 (A) Distribution of discomforts while using N-95 masks. (B) Distribution of discomforts while using N-95 masks. 
Table 1 Comparison between 6 and 8 hours of duty

\begin{tabular}{|c|c|c|c|}
\hline Duty hours & \multicolumn{2}{|c|}{$\begin{array}{l}\text { Changes in oxy- } \\
\text { gen saturation }\end{array}$} & \\
\hline \multirow[t]{4}{*}{6 hours } & No change & 8 & \multirow{10}{*}{$\begin{array}{l}\text { There is no significant } \\
\text { association between } \\
\text { the number of hours } \\
\text { and changes in oxygen } \\
\text { saturation as } z=0.73 \\
\text { and } p>0.05\end{array}$} \\
\hline & $1 \%$ decrease & 12 & \\
\hline & $2 \%$ decrease & 3 & \\
\hline & Total & 23 & \\
\hline \multirow[t]{6}{*}{8 hours } & No change & 15 & \\
\hline & $1 \%$ decrease & 21 & \\
\hline & $2 \%$ decrease & 16 & \\
\hline & $3 \%$ decrease & 3 & \\
\hline & $4 \%$ decrease & 2 & \\
\hline & Total & 57 & \\
\hline
\end{tabular}

nasal and oral routes. As we all know, various masks are available, made of various materials, but so far N-95 mask has proven most effective in controlling the spread of air-borne viral particles. Because of its thick protective covering and multiple layers, it causes discomfort to its users. The health care workers and other people working in a health care setup are the ones who have to wear this mask at all points of time as they are the ones in direct and continuous contact with potential virus carriers. Due to long hours of usage, a lot of discomforts and problems have been reported.

Suen et $\mathrm{al}^{2}$ conducted a comparative study on 104 participants at an international center for infection control, School of Nursing in Hong Kong, where they compared the fit and usability of the conventional 3M N-95 mask with the nanofiber N-95 mask. The participants wore each type of N-95 for 10 minutes and performed a nursing procedure. A comparison was made between different masks regarding their fit and usability. The nanofiber mask showed better fit and greater usability in terms of breathability, facial heat, facial pressure, speech intelligibility, itchiness, and difficulty in maintaining the mask in place, etc. Hence, the material of the $\mathrm{N}-95$ mask plays a very important role. A skin-friendly mask is more acceptable to health care workers.

With the help of a structured questionnaire, we tried to list the problems associated with wearing an N-95 mask and oxygen saturation level in the blood. The data showed that all participants had one or more discomfort associated with the mask usage. Some also showed a decrease in the oxygen saturation level in the blood, and many had decreased work efficiency after long hours of mask usage. However, even after facing many discomforts, the majority of participants were satisfied with the feeling of protection provided by the mask. Also, $50 \%$ of the participants faced breathing difficulty, $33 \%$ had mouth breathing, and $41 \%$ had itching over the face. Further study needs to be done regarding manufacturing user-friendly masks. The prolonged usage of the N-95 mask impeded the gaseous exchange and imposed an additional workload on the metabolic system of pregnant healthcare workers. ${ }^{5}$ Breathing through an N-95 mask can lead to increased carbon dioxide concentration and decreased oxygen concentration in the expired air. ${ }^{5}$

Breathing vent can solve this problem, but in the COVID pandemic, it is not advisable. Introducing a size range among the masks can prove beneficial in a better fit and probably lesser skin-related issues. According to Baig et $\mathrm{al}^{4}{ }^{4}$ the participants require a comfortable mask that does not interfere with breathing, causes less facial heat production, and can be worn with facial hair.

Modifications will make the mask more tolerable and help the health care provider deliver strenuous hospital duties with much more ease and efficacy. The COVID-19 pandemic is still prevailing, and a third wave is anticipated. Hence, timely intervention in this regard will prove beneficial in handling increased caseload with efficacy. ${ }^{6}$

\section{Conclusion}

The currently used N-95 masks are not user-friendly, and their use is associated with multiple discomforts. It can alter oxygen saturation but does not produce significant effects in healthy individuals. However, it can significantly affect patients with compromised airways. Such an observation was not made in our study because such individuals were excluded from COVID care duties. Masks with a string to tie at the back of the head are well tolerated compared with the masks with a string around the pinna. Different sizes of the mask should be made available.

\section{Ethical Approval}

This article does not contain any studies with animals performed by any of the authors. Ethical approval from the IRB was taken.

\section{Conflict of interest \\ None declared.}

\section{References}

1 Gorbalenya AE, Baker SC, Baric R, et al. Severe acute respiratory syndrome-related coronavirus: the species and its viruses-a statement of the Coronavirus Study Group. bioRxiv 2020. Doi: $10.1101 / 2020.02 .07 .937862$

2 Suen LKP, Guo YP, Ho SSK, Au-Yeung CH, Lam SC. Comparing mask fit and usability of traditional and nanofibre N95 filtering facepiece respirators before and after nursing procedures. J Hosp Infect 2020;104(03):336-343

3 Benson SM, Novak DA, Ogg MJ. Proper use of surgical n95 respirators and surgical masks in the OR. AORN J 2013;97(04):457-467

4 Baig AS, Knapp C, Eagan AE, Radonovich LJ Jr. Health care workers' views about respirator use and features that should be included in the next generation of respirators. Am J Infect Control 2010;38 (01):18-25

5 Tong PSY, Kale AS, Ng K, et al. Respiratory consequences of N95type mask usage in pregnant healthcare workers-a controlled clinical study. Antimicrob Resist Infect Control 2015;4(01):48

6 Rosner E. Adverse effects of prolonged mask use among healthcare professionals during COVID-19. J Infect Dis Epidemiol 2020; 6(03):130 